\title{
Inventory Routing Problem with Non-stationary Stochastic Demands
}

\author{
Ehsan Yadollahi ${ }^{1,2}$, El-Houssaine Aghezzaf ${ }^{1}$, Joris Walraevens ${ }^{2}$ and Birger Raa ${ }^{1}$ \\ ${ }^{I}$ Department of Industrial Systems Engineering and Product Design, \\ Faculty of Engineering and Architecture, Ghent University, Ghent, Belgium \\ ${ }^{2}$ Department of Telecommunications and Information Processing (TELIN), \\ Faculty of Engineering and Architecture, Ghent University, Ghent, Belgium
}

Keywords: Inventory Routing Problem, Stochastic Demand, Non-stationary, Optimization.

\begin{abstract}
In this paper we solve Stochastic Periodic Inventory Routing Problem (SPIRP) when the accuracy of expected demand is changing among the periods. The variability of demands increases from period to period. This variability follows a certain rate of uncertainty. The uncertainty rate shows the change in accuracy level of demands during the planning horizon. To deal with the growing uncertainty, we apply a safety stock based SPIRP model with different levels of safety stock. To satisfy the service level in the whole planning horizon, the level of safety stock needs to be adjusted according to the demand's variability. In addition, the behavior of the solution model in long term planning horizons for retailers with different demand accuracy is taken into account. We develop the SPIRP model for one retailer with an average level of demand, and standard deviation for each period. The objective is to find an optimum level of safety stock to be allocated to the retailer, in order to achieve the expected level of service, and minimize the costs. We propose a model to deal with the uncertainty in demands, and evaluate the performance of the model based on the defined indicators and experimentally designed cases.
\end{abstract}

\section{$1 \sqsubset$ INTRODUCTION}

Minimizing logistics costs has been a major issue in many industries, especially those dealing with relatively high level of costs for transportations, storage, and stock-outs (Pujawan et al, 2015). In such a situation, not only the "best" schedules for the replenishment matters, but also the estimated costs for storage capacity, holding and stock-out costs are crucial. Minimizing these costs while the promised level of service is satisfied, is the major issue in inventory routing problem.

Forecasting the expected demands is the initial requirement for Inventory Routing Problem (IRP) (Sagaert et al, 2018). The accuracy of the expected demand affects inventory level and related costs during the planning horizon. Normally these estimations are done based on the historical data gathered from previous periods. So far most of the studies about IRP have considered demands as stationary stochastic among the periods (Abdul Rahim et al, 2014; Bertazzi et al, 2015; Diaz et al, 2016; Rahim and Irwan, 2015; Yadollahi et al, 2017), while in real life cases -when the planning is done for a long horizon- the accuracy of the estimated demand may decrease among the periods and make the estimated demand more uncertain. That influences the IRP optimization in long term planning horizon regarding the minimization of the costs and covering the promised service level. A product with a random demand pattern would always have higher costs as compared to a product with sinusoidal or life cycle demand pattern from both costs and service level points of view. Therefore, a fair trade-off between service level and total costs is required (Purohit et al, 2016).

While distribution planning is considered as operational in nature, storage capacity allocation tend to be strategic (Manzini and Bindi, 2009) as they require large capital investments. Therefore, trading-off the two decisions under uncertainty is challenging. To this, we also add the non-stationarity in the stochastic demands at the retailers. In this paper first we consider solution models for Stochastic Periodic Inventory Routing Problem (SPIRP) with non-stationary demands and then reformulate it to take into account different policies for allocation of safety stock at the retailers. In the 
cases with different safety stock levels, it is important to know which model suits the best in order to allocate optimum level of inventory to minimize the costs in the whole planning horizon and still satisfy the actual demand.

\section{SAFETY STOCK-BASED SPIRP MODEL}

The distribution system studied in this paper consists of a single warehouse and a set of geographically scattered retailers. The retailers are indexed by $i$ and $j,(i, j \in\{1,2, \ldots, m\})$ where $m$ is the total number of retailers and the warehouse is indexed by $r$. Let $H=\{1,2, \ldots, T\}$ be the planning horizon covering $T$ periods each being indexed by $t$, and $H^{+}=H \cup\{0\}$ be the planning horizon that includes period $t=0$. Retailer $j$ has a demand rate $d_{j t}$ in time period $t$. Let $S$ be the set of retailers; and $S^{+}=S \cup\{r\}$.

Let $\tau$ be the size in time units of each period $t$; this can for example be the eight working hours per day. For the deliveries, a fleet of vehicles $V, v \in$ $\{1,2, \ldots, k\}$ each with a capacity of $\kappa$ is available. The supplier and each retailer $j$ agree to a service level $\left(S L_{j}\right)$ based on a predetermined stock-out probability $\theta_{j}$. This results in $S L_{j}=1-\theta_{j}$. Stockouts are assumed to be fully backlogged.

\section{Additional Parameters of the Model are as Follows:}

$\phi_{j t}$ : the fixed handling cost (in euros) per delivery at location $j \in S^{+}$(retailers and warehouse) in period $t \in H$.

$h_{j t}$ : the per unit per period holding cost of the product at location $j \in S$ (in euros per ton); $\delta_{v}$ : travel cost of vehicle $v \in V$ (in euros per $\mathrm{km})$;

$\eta_{v}$ : average speed of vehicle $v \in V$ (in km per hour);

$\Delta_{i j}$ : duration of a trip from location $i \in S^{+}$to location $j \in S^{+}$(in hours);

$I_{j 0}: \quad$ the initial inventory level at retailer $j \in S$;

The Variables of the Model are defined as Follows:

$Q_{v i j t}$ : the quantity of product in vehicle $v \in$ $V$ when it travels directly to location $j \in S^{+}$ from location $i \in S^{+}$in period $t \in H$. This quantity equals zero when the trip $(i, j)$ is not made by vehicle $v \in V$ in period $\mathrm{t}$;

$q_{j t}:$ the quantity delivered to location $j \in S$ in period $t \in H$;

$I_{j t}$ : the inventory level at location $j \in S$ by the end of period $t \in H$;

$x_{v i j t}$ : a binary variable set to 1 if location $j \in S^{+}$ is visited immediately after location $i \in S^{+}$ by vehicle $v \in V$ in period $t \in H$, and 0 otherwise;

$y_{v t}$ : a binary variable set to 1 if vehicle $v \in V$ is being used in period $t$, and 0 otherwise;

The optimization problem we face is the following;

Minimize:

$\psi_{v}:$ the fixed operating cost of vehicle $v \in V$ (in euros per vehicle);

$$
C V=\sum_{t \in H} \sum_{v \in V}\left[\psi_{v} y_{v t}+\sum_{i \in S^{+}} \sum_{j \in S^{+}}\left(\delta_{v} \eta_{v} \Delta_{i j}+\phi_{j t}\right) x_{v i j t}\right]+\sum_{t \in H} \sum_{j \in S} h_{j t} I_{j t}
$$


Subject to:

$$
\begin{gathered}
\sum_{v \in V} \sum_{i \in S^{+}} x_{v i j t} \leq 1, \forall j \in S, t \in H \\
\sum_{i \in S^{+}} x_{v i j t}-\sum_{k \in S^{+}} x_{v j k t}=0 \forall j \in S^{+}, t \\
\in H, v \in V
\end{gathered}
$$

$$
\sum_{i \in S^{+}} \sum_{j \in S^{+}} \Delta_{i j} x_{v i j t} \leq \tau_{t} \forall t \in H, v \in V
$$

$$
\sum_{v \in V} \sum_{i \in S^{+}} Q_{v i j t}-\sum_{\substack{v \in V \\ \in S, t \in S^{+}}} \sum_{v j k t}=q_{j t} \forall j
$$

$$
Q_{v i j t} \leq \kappa x_{v i j t}, \forall i \in S^{+}, j \in S^{+}, t \in H, v
$$

$$
\begin{array}{r}
I_{j 0} \leq I_{j T}, \quad \forall j \in S, t \in H \\
x_{v r j t} \leq y_{v t}, \forall j \in S^{+}, t \in H, v \in V \\
x_{v i j t}, y_{v t} \in\{0,1\}, I_{j t} \geq 0, Q_{v i j t} \geq 0, q_{j t} \geq 0, \\
\forall j \in S^{+}, t \in H, v \in V
\end{array}
$$

The objective function (1) shows the variables to minimize the level of costs in this replenishment system. It includes five cost components, namely, total fixed operating cost of using the vehicle(s), total transportation cost, total delivery handling cost, total inventory holding cost at the end of each period.

Constraints (2) assure that each retailer is visited at most once during each period. Constraints (3) guarantee that a vehicle moves to the next retailer/depot after serving the current one.
Constraints (4) prevent that the time required to complete each tour does not exceed the duration of the period. The quantities to be delivered to each retailer are determined by constraints (5). These constraints also avoid sub-tour(s) from occurring. Constraints (6) are capacity constraints induced by the vehicles capacities. Constraints (7) determine the delivered number of products from period 1 to $t$ together with the initial inventory to be equal to the expected demand's values from period 1 to $t$, safety stock, and remaining inventory at the end of period $t$ for each retailer $j$. Constraints (8) insure that the level of inventory at the end of last period is equal or larger than initial inventory. Finally, constraints (9) specify that a vehicle cannot be assigned to serve retailers unless the related fixed cost is payed.

\subsection{Safety Stock based SPIRP}

Safety stock is a term used by logisticians to describe a level of extra stock that is maintained to diminish risk of stock-outs caused by uncertainties in supply and demand. It is an additional quantity of an item held on top of the cycle inventory to reduce the risk that the item will be out of stock. The amount of safety stock and its allocation mechanism during short/long term planning horizon is considered in this section. This approach reformulates the SPIRP to a safety stock-based equivalent deterministic model, where extra amount of stock is kept at retailers to cope with their demands' variability.

This approach can be seen as an application of Robust Optimization. Bertsimas et al (2011) formulated the optimization model under uncertainty to a deterministic equivalent one. The proposed approximate deterministic model in this section is a robust reformulation of SPIRP and reformulates the model to a safety stock-based deterministic equivalent.

As is presented in table 1 , safety stock is a function of service level parameter $\left(z_{\theta_{j}}\right)$, number of time periods $(t)$, and standard deviation of demand $\left(\sigma_{j t}\right)$ for each retailer $(j)$. The parameter $z_{\theta_{j}}$ is the service factor determined by retailer's requested service level $\left(S L_{j} \%\right)$ gained by the level of $\theta_{j}$ as the inventory violation rate. It is used as a multiplier with the standard deviation and number of time periods to calculate a specific quantity (as safety stock) to meet the pre-set service level. 
Table 1: Safety Stock models.

Safety Stock allocation mechanism

\begin{tabular}{|c|c|c|c|}
\hline Model 1 & $S S_{j t}=z_{\theta_{j}} \sum_{l=1}^{t} \sigma_{j l}$ & Model 6 & $S S_{j t}=\frac{z_{\theta_{j}}}{4 t} \sum_{l=1}^{t} \sigma_{j l}$ \\
\hline Model 2 & $S S_{j t}=\frac{z_{\theta_{j}}}{\sqrt{t}} \sum_{l=1}^{t} \sigma_{j l}$ & Model 7 & $S S_{j t}=\frac{z_{\theta_{j}}}{5 t} \sum_{l=1}^{t} \sigma_{j l}$ \\
\hline Model 3 & $S S_{j t}=\frac{z_{\theta_{j}}}{t} \sum_{l=1}^{t} \sigma_{j l}$ & Model 8 & $S S_{j t}=\frac{z_{\theta_{j}}}{10 t} \sum_{l=1}^{t} \sigma_{j l}$ \\
\hline Model 4 & $S S_{j t}=\frac{z_{\theta_{j}}}{2 t} \sum_{l=1}^{t} \sigma_{j l}$ & Model 9 & $S S_{j t}=\frac{z_{\theta_{j}}}{t^{2}} \sum_{l=1}^{t} \sigma_{j l}$ \\
\hline Model 5 & \multicolumn{3}{|c|}{$S S_{j t}=\frac{z_{\theta_{j}}}{3 t} \sum_{l=1}^{t} \sigma_{j l}$} \\
\hline
\end{tabular}

\section{CASE STUDY}

We consider a distribution center with one retailer and one warehouse. There is one vehicle with the capacity of $200 \mathrm{~kg}$. The vehicle works 8 hours per day with an average speed of $50 \mathrm{~km} / \mathrm{h}$. Fix and variable costs of the vehicle are presented in table 3 . Distance between the retailer and warehouse is about $25 \mathrm{~km}$ and it takes 0.5536 hour. The demand for the retailer is considered stochastic and follows Gamma distribution and all the stock-outs are fully backlogged. Table 2 presents the demands for 1 period time and standard deviations as well as their coefficient of variations. The rest of the parameters of this example are provided in table 1 . We use CPLEX 12.5.1 for solving all models. All the computations are performed on a $3.60 \mathrm{GHz}$ Intel® Xeon ${ }^{\circledR}$ CPU.

\subsection{Design of Experiments}

The illustrative example consists of one retailer and one warehouse to simplify the routing optimization and put the emphasize more on the inventory management. We take into account different instances with different demands and planning horizons. The detail of the experimental design is presented below:

\section{Safety Stock Allocation Model.}

There are 9 considered models to allocate safety stock to the retailer (table1).

\section{Planning Horizon.}

50 periods.

\section{Demand's Accuracy Level.}

The accuracy level shows the growing uncertainty among the periods. In this example we considered 5 different levels presented in table 2 .

In total there are 45 instances considered in this instance. The outcome of the optimization models are simulated, compared and analysed in next section.

\subsection{Non-stationary Demands}

The stochastic demand we consider is nonstationary, which means its distribution varies from one period to the next. Demand in period $t$ is represented by means of a non-negative random variable $\left(d_{j t}\right)$ with known cumulative distribution function $F_{t}$ : Random demand is assumed to be independent over the periods. The idea is to figure out the most optimum way of allocating safety stocks at the retailer with different standard deviations among the periods. In table 2 the averages and standard deviations of the demand for the considered retailer are presented. $x_{j}$ is the certainty rate multiplied by the standard deviation of the demands, showing the influences during the planning horizon on the estimated demand. 
Table 2: Retailer's Demands.

Average

Retailer
Uncertainty level (Standard deviation)

$E\left(d_{j t}\right)=21.98$ $\sigma_{j t}=\left(1-x_{j}\right) t$
Accuracy rate

$\left(\boldsymbol{x}_{j}\right)$

$99 \%, 98 \%, 95 \%, 90 \%$, and $80 \%$
Retailer's demand follows Gamma distribution $\Gamma\left(\alpha_{j t}, \beta_{j t}\right)$. Since the demands are non-stationary, the parameters for Gamma distribution are dependent on $t$. According to the defined trends $\left(x_{j}\right)$ for the demand at each retailer, $\alpha_{j t}$ and $\beta_{j t}$ take different values.

In this paper first we do the experiment without involving the entropy level, just to see how different models behave, and then we add the entropy effect $\left(x_{j}\right)$ on safety stock calculation to check with the results. Of course the results should be better, but we measure whether the indicators are improved.

Table 3: Parameter values.

\begin{tabular}{|c|c|c|}
\hline Notation & Parameter & values \\
\hline$\phi_{j t}$ & Handling costs & 25 \\
\hline$\eta_{j t}$ & $\begin{array}{l}\text { Inventory holding costs per unit } \\
\text { per period }\end{array}$ & 0.5 \\
\hline$\delta_{v}$ & $\begin{array}{l}\text { Travel costs for vehicle in Euro } \\
\text { per KM }\end{array}$ & 1 \\
\hline$\psi_{v}$ & Fix operating cost of vehicle & 30 \\
\hline$v_{v}$ & Average speed of vehicle & 50 \\
\hline
\end{tabular}

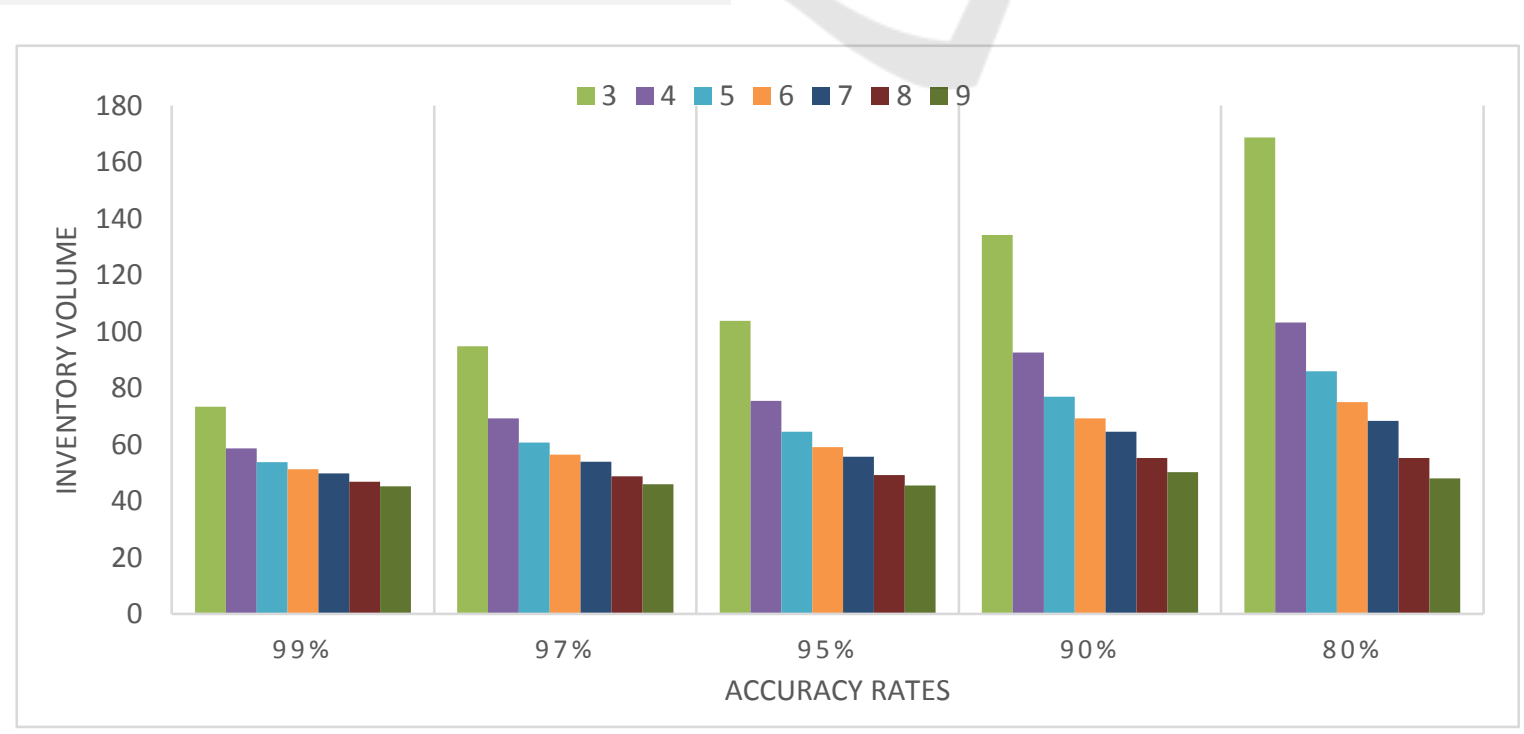

Figure 1: Inventory level.

\section{RESULTS AND DISCUSSIONS}

The instances of DOE have been optimized and simulated for 280 replications. The simulation model generates gamma distributed demands according to $\alpha_{j t}$ and $\beta_{j t}$. The optimized results show the amount of delivered product to the retailer in different periods, together with inventory level at each period. Also the costs to expect from this model. To verify this, we simulated the DOE instances 280 times and compared the results with the estimated outcome from optimization model.

The indicators chosen in this paper show an interesting move amongst different instances. Figure 1 displays the average inventory levels at the end of the planning horizon for each instance. The horizontal axis shows the accuracy rates of our data, to see whether the inventory level changes if the provided data is not accurate. As it is shown in this figure, the level of inventory increases slightly when the data accuracy is decreasing.

In addition, different considered safety stock based model have different effects on the inventory level. Model 9 (table 1) has the lowest inventory while Model 1 has the highest volume. This

ory level. 
difference is because of the safety stock reduction policy in long term planning horizons. Moreover, the difference between different models is the lowest when the accuracy is $99 \%$, meaning all models behaving similar when the certainty of the demand rate is the highest. By having low accuracy, the models need to allocate more inventory to the retailer and that results in high end inventory level. The other important indicator in this study is inventory violation. This indicator shows the percentage of having the retailer out of stock during the whole planning horizon. These percentages are shown in figure 2 for all the instances considered in this paper. In this cases we pre-defined $10 \%$ of inventory violation among the planning horizon, and according to this, we check whether the actual stockout level varies in different instances.
The horizontal axis in figure 2 displays the accuracy rates of different instances, while the vertical axis shows the backlog percentage. As it is shown in this figure, lack of accuracy in data results in minor changes in IV levels. Even-though the accuracy level is around $80 \%$, still the models are able to cover the demands for more than $82 \%$ in the worst case (model 9), and $100 \%$ in the best cases (model 1,2, and 3). The trend in different accuracy rates is the same. Model 1 is always with no stockout and model 9 with high stock-out level.

To have a better understanding of this indicator, figure 3 presents the differences between expected and actual level of backlog. Positive values demonstrate the model satisfaction of the estimated guaranteed service level and negative values show the failure of the models to cover the estimated level

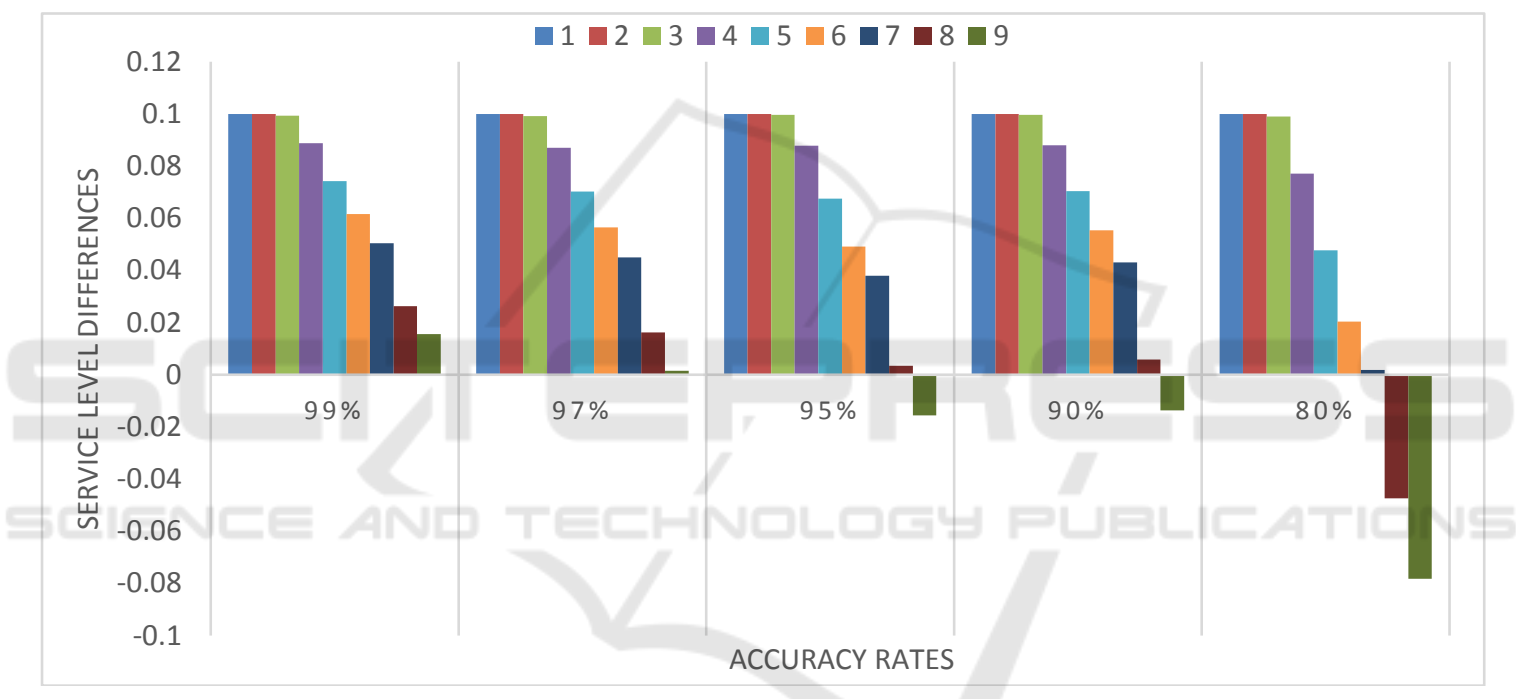

Figure 2: Service Level Accuracy.

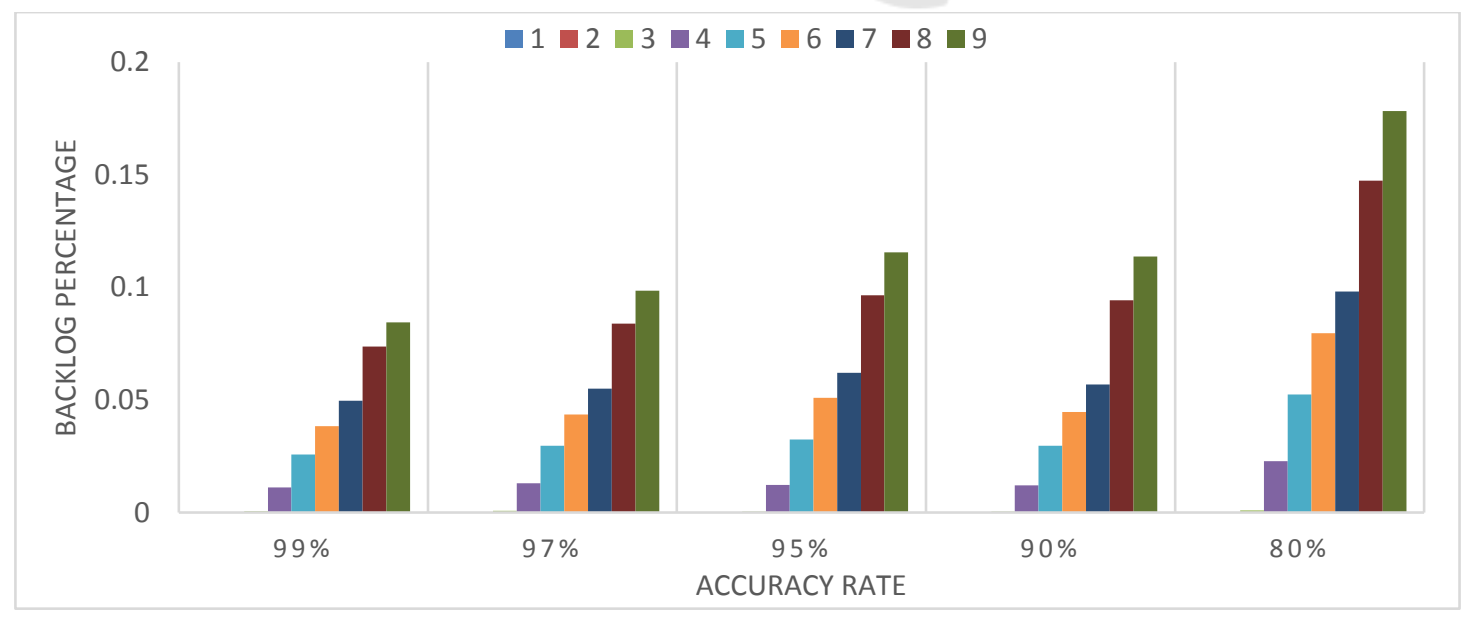

Figure 3: Stock-out level. 


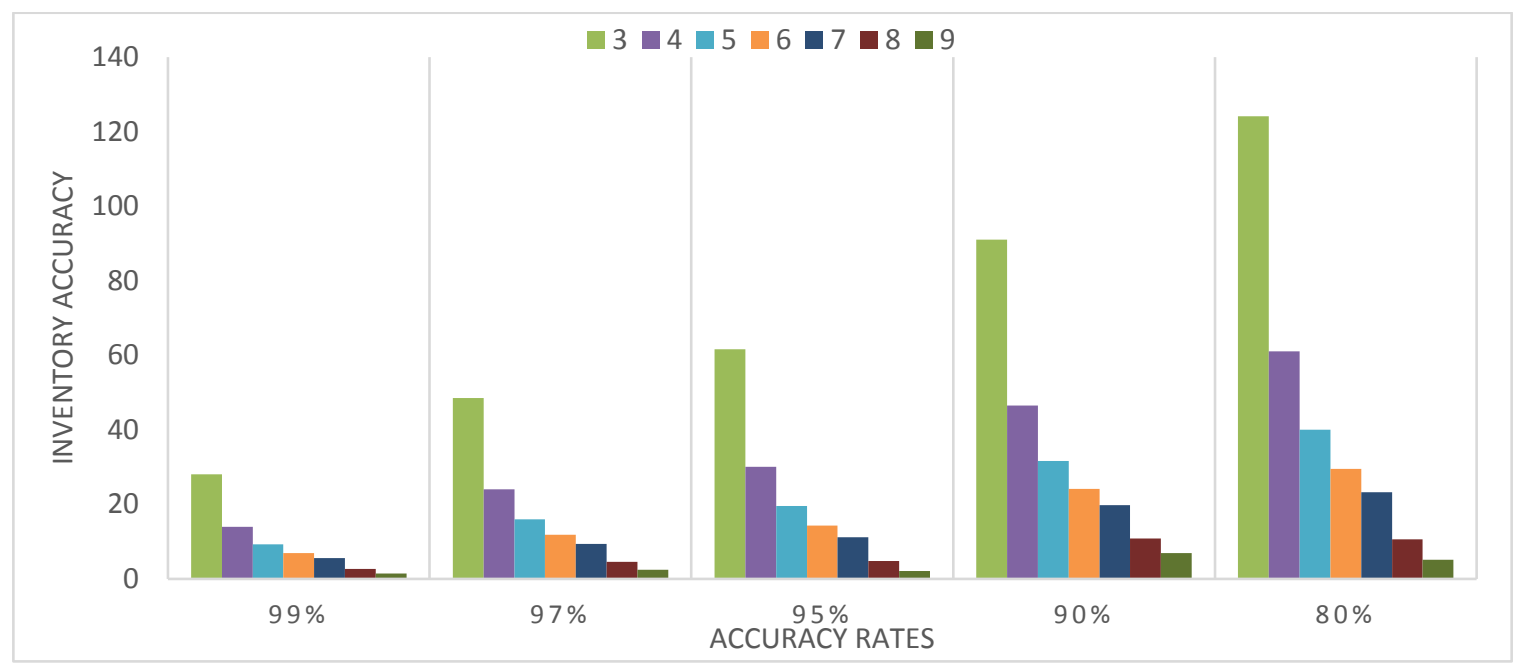

Figure 4: Inventory Accuracy.

of service. As it is clear, all the instances satisfied the expected service except model 9 for the cases with 95, 90, and 80 percent accuracy in data and model 8 for the case with 80 percent accuracy in data. This figure clarifies the ability of the proposed models in satisfying the demands in different situations. Even-though the amount of safety stock decreases by the number of proposed models, still they manage to have the expected service level.

From the other side this figure shows that in most of the cases the actual level of service is more than what was expected (more than 90 percent while $90 \%$ is enough), which means that the retailer keeps extra level of inventory in most of the periods of planning horizon to deal with the uncertainty in demands. Therefore, having the bars more close to zero in figure 3 shows the efficiency of the model (in this case model 8) in satisfying the demand while avoiding the huge inventory level.

To check with the models to see whether they are accurate in their results, we compare the estimated level of inventory at each period with actual levels. Figure 4 illustrates this differences for all the considered cases in this paper. Models with lower level of safety stock are more accurate in the inventory level in comparison with the ones with bigger safety stock (we have excluded model 1 and 2 (table 1) in this figure due to the high level of difference in inventory). In addition, the cases with lower data accuracy have lower accuracy in their results which makes sense, because the model needs to compensate it with more delivered products which might not be used in the end.

\section{CONCLUSION}

In this paper we considered stochastic demands in IRP when the variability of demand increases among the periods. Several uncertainty rates are examined as well as different safety stock-based models to solve the SPIRP model. We developed the SPIRP model for one retailer with an average level of demand, and standard deviation for each period. The objective is to find an optimum level of safety stock to be allocated to the retailer, in order to achieve the expected level of service, and minimize the costs. The performance of the model based on the defined indicators and DOE cases is evaluated for a 50 period planning horizon, and simulated for 280 replications to compare the expected results with actual outcomes.

The results have shown a gradual reduction in inventory levels at the retailer for the cases with smaller safety stock level. The models 7, 8, 9 (table 1) are almost the same regarding the inventory volume and accuracy check, among all the defined uncertainty levels. These models showed that for the long term planning horizon we are able to reduce the safety stock to minimize the costs. In addition, in these models the impact of uncertainty level is less than other models. Expected service level is achieved in all the scenarios except for some cases of model 9 and one case of model 8, due to the lack of available inventory. For the future research, we will involve more variation of cases in the design of experiments to be able to evaluate the model from different perspectives. 


\section{REFERENCES}

Abdul Rahim, M. K. I., Zhong, Y., Aghezzaf, E.-H. and Aouam, T. (2014) Modelling and solving the multiperiod inventory-routing problem with stochastic stationary demand rates. International Journal of Production Research, 52(14), 4351-4363.

Bertazzi, L., Bosco, A. and Laganà, D. (2015) Managing stochastic demand in an Inventory Routing Problem with transportation procurement. Omega, 56, 112-121.

Bertsimas, D., Brown, D. B. and Caramanis, C. (2011) Theory and Applications of Robust Optimization. SIAM Review, 53(3), 464-501.

Diaz, R., Bailey, M. P. and Kumar, S. (2016) Analyzing a lost-sale stochastic inventory model with Markovmodulated demands: A simulation-based optimization study. Journal of Manufacturing Systems, 38, 1-12.

Manzini, R. and Bindi, F. (2009) Strategic design and operational management optimization of a multi stage physical distribution system. Transportation Research Part E: Logistics and Transportation Review, 45(6), 915-936.

Pujawan, N., Arief, M. M., Tjahjono, B. and Kritchanchai, D. (2015) An integrated shipment planning and storage capacity decision under uncertainty A simulation study. International Journal of Physical Distribution and Logistics Management, 45(9-10), 913-937.

Purohit, A. K., Shankar, R., Dey, P. K. and Choudhary, A. (2016) Non-stationary stochastic inventory lot-sizing with emission and service level constraints in a carbon cap-and-trade system. Journal of Cleaner Production, $113,654-661$.

Rahim, A. and Irwan, M. K. (2015) On the inventory routing problem with stationary stochastic demand rate. Ghent University.

Sagaert, Y. R., Aghezzaf, E.-H., Kourentzes, N. and Desmet, B. (2018) Tactical sales forecasting using a very large set of macroeconomic indicators. European Journal of Operational Research, 264(2), 558-569.

Yadollahi, E., Aghezzaf, E. H. and Raa, B. (2017) Managing inventory and service levels in a safety stock - based inventory routing system with stochastic retailer demands. Applied Stochastic Models in Business and Industry. 